\title{
Novas Perspectivas na Gestão do Sistema de Saúde da Espanha
}

\section{New Perspectives on the Management of the Spanish National Health System}

\author{
Daniele Pompei Sacardo \\ Doutora em Saúde Pública. \\ Endereço: Av. Dr. Arnaldo, 715, Cerqueira César, CEP 01246-904, \\ São Paulo, SP, Brasil. \\ E-mail: danipsacœusp.br

\section{Paulo Antonio de Carvalho Fortes} \\ Prof. Titular da Faculdade de Saúde Pública da Universidade de \\ São Paulo \\ Endereço: Av. Dr. Arnaldo, 715, Cerqueira César, CEP 01246-904, \\ São Paulo, SP, Brasil. \\ E-mail: pacfuspœusp.br

\section{Oswaldo Yoshimi Tanaka} \\ Prof. Titular da Faculdade de Saúde Pública da Universidade de \\ São Paulo \\ Endereço: Av. Dr. Arnaldo, 715, Cerqueira César, CEP 01246-904, \\ São Paulo, SP, Brasil. \\ E-mail: oytanakaœusp.br
}

\section{Resumo}

Ao longo dos últimos vinte e cinco anos, a organização do sistema de saúde na Espanha vem adotando diversas medidas que reorientaram seu gerenciamento, melhoraram sua eficiência e aprimoraram seu sistema de financiamento, resultado de profundas reformas e da introdução de novos instrumentos de gestão. Este artigo é resultado de uma análise documental que objetivou descrever a trajetória de conformação do sistema de saúde espanhol e sua organização na contemporaneidade. Apresenta alguns determinantes históricos que tornaram possíveis as reformas no setor sanitário, como a descentralização para o nível das Comunidades Autônomas, a incorporação de mecanismos de coordenação e a integração e o financiamento dos novos e distintos formatos organizativos coexistentes no país. Além disso, identifica desafios que emergem no cenário atual do Sistema Nacional de Saúde, como o fenômeno da imigração, o avançado processo de transição demográfica, a crescente demanda por melhorias na qualidade da atenção e de incorporação tecnológica. Todos esses fatores influem na sustentabilidade do sistema, o que motivou a criação de mais um espaço para estabelecimentos de consensos sobre o papel fundamental do sistema sanitário para o Estado de Bem-Estar espanhol.

Palavras-chave: Sistemas de Saúde; Políticas de saúde; Reformas sanitárias; Modalidades de gestão. 


\section{Abstract}

During the last twenty-five years, the organization of the health system in Spain has adopted diverse measures that reoriented its management and improved its efficiency and financing system as a result of deep reforms and introduction of new management instruments. This paper is a result of a documental analysis that aimed to describe the trajectory of conformation of the Spanish health system and its current organization. The paper presents some historical determinants that enabled the reforms in the sanitary sector, such as the decentralization to the Autonomous Communities level, the incorporation of coordination mechanis$\mathrm{ms}$, and the integration and financing of the new and distinct organizational formats that coexist in the country. Furthermore, it identifies challenges that emerge in the current scenario of the National Health System, as the immigration phenomenon, the advanced process of demographic transition, and the growing demand for improvements in the quality of care and of technological incorporation. All these factors influence the sustainability of the system, which motivated the creation of one more space for the establishment of a consensus on the fundamental role of the sanitary system for the Welfare State in Spain.

Keywords: Health Care Systems; Health Policies; Health Systems Reforms; Management Arrangements.

\section{Introdução}

Nos últimos anos, gestores e autoridades sanitárias da Espanha têm buscado experimentar novas abordagens e adotar diferentes instrumentos na gestão dos serviços de saúde, considerando os desafios impostos pelo processo de transição epidemiológica e demográfica que vem ocorrendo rapidamente nesse país. A especificidade da trajetória do sistema sanitário espanhol tem sido amplamente analisada por diversos autores (Del Aguila e Montoro, 1984; Almeida, 1995; Etviti e Leyva, 1995), que destacam a busca por consensos e pactos ao redor de questões que pudessem viabilizar e fortalecer a democracia no processo de negociação política.

Este artigo é resultado de uma análise documental que objetivou descrever a trajetória de conformação do sistema de saúde espanhol, analisar seu percurso histórico e a organização do sistema na contemporaneidade. Como caminho metodológico adotou-se a pesquisa de cunho qualitativo, utilizando-se o método descritivo-comparativo. Esse método, muito utilizado nas ciências humanas, que necessitam recuperar dados históricos para explicar a realidade social de determinado fenômeno, considera o processo tanto ou mais importante do que o produto ou o resultado. Marconi e Lakatos (2008) informam que o método descritivo geralmente é usado quando se pretende buscar o conhecimento de determinadas informações a partir da descrição de fatos e fenômenos da realidade investigada. Tendo como base o Sistema Único de Saúde (SUS) brasileiro, o método comparativo permitiu analisar dados concretos, deduzindo deles os elementos constantes, abstratos e gerais. De acordo com Gil (1994), essa abordagem consiste em levantar dados e informações embasadas em bibliografia especializada sobre conceitos teóricos e em documentos históricos, possibilitando analisá-los de modo aprofundado, perceber regularidades, identificar continuidades e descontinuidades, deslocamentos e transformações.

Ao longo da pesquisa, foram selecionados documentos oficiais, relatórios com autoria conhecida, pareceres técnicos e publicações disponíveis no acervo digital do Ministério da Saúde da Espanha, 
disponíveis em página eletrônica ${ }^{1}$ e em bibliotecas brasileiras. Todas as publicações consultadas se encontram devidamente referenciadas na bibliografia que compõe este artigo.

O atual sistema de saúde público da Espanha desenvolveu-se a partir do final do período de guerra civil com a criação, em 1942, dos "Seguros Obligatorios de Enfermedad” (Ponsá e col., 2003), que proporcionavam assistência médica a aproximadamente 50\% da população, oferecendo proteção apenas a trabalhadores industriais, tendo sua cobertura, paulatinamente, ampliada a trabalhadores manuais, a empregados em geral e finalmente à classe média. A prestação de serviços de saúde, nessa época, estava vinculada à Seguridade Social, subordinada aos Ministérios do Trabalho e da Seguridade Social, assim permanecendo até 1977.

A organização dos serviços de saúde caracterizava-se por uma extensa rede de centros e instituições próprias de caráter ambulatorial e hospitalar, porém escasso desenvolvimento de outras redes assistenciais, propiciando, dessa forma, forte integração de financiamento e produção ou provisão dos serviços. As instituições sanitárias públicas assim configuradas organizavam-se de forma vertical para o desempenho de suas atividades, eram regidas pelo princípio de hierarquização, submetidas ao Direito Público em todos seus âmbitos e sujeitas a forte processo de centralização na tomada de decisões (Martín, 1998).

No final da década de 1970, cerca de $80 \%$ das fontes de financiamento do sistema de saúde eram cotizações, demonstrando a significativa dependência da assistência sanitária dos recursos da Seguridade Social (Almeida, 1995).

A partir de 1975, a situação de expansão da Seguridade Social transformou-se radicalmente devido às restrições fiscais do Estado, que passou a priorizar a racionalização do sistema. Na esfera política, com a morte do general Franco, em 1975, iniciou-se o processo de transição política e, concomitantemente, a conformação de acordos, conhecidos como "Pactos de Moncloa", entre os distintos atores compromissados com a democratização do país, (Martín, 1998).
A Constituição de 1978 estabeleceu novo regime político pautado na organização territorial em $\mathrm{Co}$ munidades Autônomas (CCAA) com autonomia para a elaboração de estatutos regionais específicos. Em relação à Seguridade Social, a Carta Constitucional padronizou e universalizou o direito aos serviços de proteção social, inclusive a assistência sanitária, instituiu a descentralização do sistema e a devolução às CCAA dos serviços até então centralizados no Estado. Além disso, criou duas novas entidades gestoras - o Instituto Nacional de Seguridad Social (INSS) e o Instituto Nacional de Salud (Insalud), separando a seguridade social da assistência sanitária. No âmbito da saúde, o Insalud constituía o organismo federal responsável pela gestão direta dos serviços de saúde no território espanhol e era organizado em três níveis de assistência: primária, especializada extra-hospitalar e hospitalar (Rey Del Castillo, 1998).

\section{O Processo de Reforma e a Coordenação Sanitária}

Com a democracia, atores sociais progressistas propugnavam uma reforma sanitária baseada no conceito de Serviço Nacional de Saúde, com cobertura universal e financiado com impostos estatais. Os "Pactos de Moncloa" (Martín, 1998) possibilitaram o financiamento da saúde por meio, fundamentalmente, de impostos, ainda que não equivalessem a lei. A aprovação da Lei Geral da Saúde, em 1986, foi marcada por conflitos entre os diferentes atores envolvidos no processo, que contrariavam interesses de boa parte dos médicos, temerosos de perder seus privilégios, e por outros setores econômicos ligados à saúde - indústria farmacêutica, empresas hospitalares -, que vislumbravam um futuro incerto para seus negócios (Fernández-Cuenca, 1998).

Uma das funções do Estado definida pela Constituição de 1978 e desenvolvida na Lei Geral da Saúde refere-se à coordenação geral do sistema de saúde espanhol. Com essa lei, ocorreu uma mudança na legislação do sistema sanitário, cuja principal característica, inicialmente, foi a multiplicidade

1 http://www.msc.es, último acesso em 28 de abril de 2009. 
de redes públicas com predomínio da rede estatal associada à Seguridade Social, passando para um modelo sanitário territorial - Sistema Nacional de Saúde, que se configurava num conjunto de serviços de saúde coordenados entre si, integrando a assistência sanitária às distintas redes públicas de saúde, sob responsabilidade principal das Comunidades Autônomas.

O processo de descentralização do sistema sanitário do Insalud para as Comunidades Autônomas ocorreu de forma lenta e desigual. Rey Del Castillo (1998) descreve que, desde sua origem, a descentralização foi motivada por razões políticas e ocorreu paralelamente ao processo de reorganização do Estado. Entre a atribuição de responsabilidades às Comunidades Autônomas (por volta de 1980), a vigência da Lei Geral da Saúde - na qual os critérios de funcionamento geral do sistema sanitário foram determinados -, e sua efetiva implementação (até 1994), as transferências sanitárias incluíram o planejamento e a transferência de centros de saúde e de redes específicas, tendo sido a Catalunha a primeira província do país a receber transferências.

$\mathrm{O}$ autor destaca diferenças marcantes em relação à temporalidade para transferir competências desde o nível central para algumas regiões, como a Comunidade Valenciana, Navarra e Canárias. Nos três casos, a possibilidade de assumir a gestão da saúde pública teve iníciou em 1982, porém cada uma das províncias mencionadas assumiu a gestão de seus serviços de saúde em temporalidades distintas, resultando em dificuldades relevantes no que se refere à coordenação e funcionamento, provocados pela lentidão da descentralização, pela existência de diferentes sistemas organizativos e pela manutenção da estrutura do Insalud em grande parte do território nacional. Em quase todas as CCAA, constituiu-se o Serviço Regional de Saúde, que fez a gestão de seu próprio serviço assistencial articulando-os com os oferecidos pelo Insalud (Rey Del Castillo, 1998).

A coexistência de regiões relativamente autônomas e outras dependentes da administração central configurou-se, até o final dos anos 1990, num mosaico de situações que caracterizaram uma fragmentação do sistema sanitário espanhol. As funções correspondentes ao Ministério da Saúde, como autoridade sanitária no nível nacional responsável por planejar e coordenar o funcionamento do SNS, principalmente o seu financiamento, não estavam claramente definidas na perspectiva das CCAA. Estas não reconheciam o Ministério da Saúde como autoridade nacional com legitimidade institucional para implementar políticas para, efetivamente, aumentar e garantir a equidade e a qualidade do funcionamento do sistema (Durán e col., 2006).

0 processo de descentralização das competências sanitárias na Espanha levou mais de vinte anos para se completar (entre 1981 e 2004), tendo como marco legal a Lei de Coesão e Qualidade do Sistema Nacional de Saúde, promulgada em maio de 2003, que garante a coordenação e a cooperação dos distintos Serviços Regionais. Com esse processo efetivado, a melhoria da gestão em diversas CCAA em relação à acessibilidade aos serviços de saúde e à incorporação de novas tecnologias pôde ser percebida.

\section{o Sistema Nacional de Saúde e as Novas Formas de Gestão Sanitária}

O Sistema Nacional de Saúde é baseado nos princípios de igualdade no acesso, na equidade das políticas de saúde, que devem estar orientadas para a superação dos desequilíbrios territoriais e sociais, devendo suas ações priorizar a promoção da saúde e a prevenção das enfermidades. Além disso, o Sistema Nacional de Saúde responsabiliza-se pelo financiamento das Administrações Públicas, fundamentalmente por meio de impostos, e garante mecanismos de participação comunitária nos distintos níveis organizativos (Durán e col., 2006).

Um aspecto significativo de mudança organizacional foi a transformação do modelo de assistência com somente dois níveis: Atenção Primária e Atenção Especializada. A integração das funções de promoção da saúde e prevenção de doenças (responsabilidade das CCAA nas funções de saúde pública) com as funções assistenciais (desenvolvidas em grande parte do país pelo Insalud até o ano de 2002) não foram alcançadas plenamente nos anos 1980, o que desencadeou um processo de redimensionamento dos esquemas de competências dos distintos níveis de atenção e de gestão. Martín assinala que o processo de inovação institucional e organizacional 
dos sistemas sanitários ocorreu paralelamente a um processo mais amplo e global de reorientação do Estado de Bem-Estar e suas formas de arranjo e gestão. Conforme o autor, o êxito desses processos de inovação foi a chave para estabelecer as linhas de defesa do Estado de Bem-Estar diante das estratégias de "liberalismo radical" que veem no mercado a solução de todos os problemas.

A modalidade dominante no sistema sanitário espanhol é a Gestão Direta sem personalidade jurídica própria. Nesse modelo, os centros sanitários respondem aos princípios e às restrições gerais do setor público em seu funcionamento e organização. A Administração Pública fiscaliza por meio da "Intervenção" o cumprimento da legalidade vigente, o pessoal é estatutário e o sistema de alocação de recursos, segmentado, ou seja, com orçamentos separados para a Atenção Primária e a Atenção Especializada. O cumprimento da normativa é o principal objetivo dessa modalidade de Administração, não raro em detrimento da eficiência na utilização dos recursos alocados.

Ainda assim, novas formas de gestão dos serviços de saúde foram implementadas, inicialmente em caráter experimental, nos novos hospitais para dotálos de personalidade jurídica própria com o intuito de compará-los com outras modalidades de gestão e avaliar suas repercussões nos diferentes aspectos. Paralelamente, a Gestão Indireta desenvolveu um conjunto de novas iniciativas, entre as quais vale destacar a figura da concessão administrativa, as entidades de base associativa e os acordos de caráter substitutivo (Ventura, 1998).

Conceitualmente, como descreve Martín (1998), as novas experiências em gestão sanitária na Espanha estão marcadas pelo "paradigma" da denominada "competência gestionada", cuja ideia central é separar o financiamento e a regulação da provisão e produção de serviços. Os financiadores, mediante contratos, destinariam recursos aos provedores sanitários transferindo-lhes certo grau de riscos e, portanto, de potenciais benefícios e perdas. Os principais argumentos apresentados para desencadear o "processo de inovação" foram a importância da autonomia dos hospitais e outros provedores sanitários, o desenvolvimento da gestão clínica, a orientação dos serviços para a qualidade, a recuperação do protagonismo dos usuários e a ênfase crescente na efetividade da prática clínica, adotando-se protocolos pautados na medicina baseada em evidências.

Os esquemas de "competência gestionada" foram articulados e formulados no nível político a partir do "Informe Abril" (Martín, 1998) que, além de propor a separação das funções de financiamento, compra e provisão de serviços, criou um mercado interno de provedores com a finalidade de estimular a competição e sugeriu que os centros de saúde passassem a se configurar como empresas públicas regidas pelo direito privado, portanto, fora do âmbito do direito administrativo. Esse processo de transformação institucional e organizativo, que atentasse às propostas de novas formas de gestão, foi, todavia, bastante lento, salvo no Estado da Catalunha, pioneira no desenvolvimento de modelos inovadores de gestão sanitária.

A prestação e a gestão dos serviços sanitários ou sociossanitários pôde ocorrer, além dos meios próprios, mediante acordos, convênios ou contratos com pessoas ou entidades públicas ou privadas, nos termos previstos na Lei Geral da Saúde.

Apresentam-se, a seguir, algumas modalidades de gestão sanitária em curso atualmente relatadas pelo Observatório do Sistema Nacional de Saúde espanhol (España, 2007), que já possuem estudos e avaliações que evidenciam serem mais eficientes comparativamente ao modelo burocrático "tradicional”.

\section{Mecanismos de Gestão Direta}

Esta modalidade adotou o "contrato-programa”, que segue o modelo de direção por objetivos mediante o qual se pactuam os objetivos e recursos disponíveis. Os contratos-programas desencadearam melhorias no monitoramento e avaliação do produto sanitário, porém sofreram alguns problemas como a falta de penalidade no caso de não cumprimento e exigências legais rígidas, como o controle e a alocação do gasto em áreas específicas, o que restringia a capacidade de gestão efetiva dos gestores/gerentes.

Fundações Públicas Hospitalares: caracterizam-se por entidades com personalidade jurídica própria, regidas por um diretivo controlado pelas autoridades públicas sanitárias, que determinam 
um rol de atividades a serem contratadas. Uma questão importante de ser esclarecida nesse caso é a eficácia alcançada com a autonomia e a contratação de pessoal diante dos problemas de controle efetivo por parte das autoridades sanitárias quando se introduzem mais níveis hierárquicos na estrutura organizativa.

Outra modalidade de Gestão Direta alternativa ao modelo burocrático é a criação de Empresas Públicas Sanitárias, que podem ser organizadas de duas maneiras: Entidade de Direito Público, cuja atividade se sujeita ao direito privado e as Empresas Públicas com forma societária, cuja titularidade corresponde a uma Administração Pública. Algumas características organizativas são a autonomia na gestão dos recursos humanos que possibilita um sistema de seleção de candidatos baseado no perfil de competências e na adaptação à cultura local. Um sistema de retribuição que combina um salário fixo baseado na categoria e outra quantia variável baseada no alcance de objetivos. A autonomia financeira também possibilita à Administração Pública gerenciar seus recursos, o que implica uma maior flexibilidade e capacidade de concentração nas questões de atenção. 0 sistema de controle da gestão baseia-se no contrato programa com o Conselho de Saúde, mediante o qual se fixa a quantidade de atividades a ser realizada e o orçamento. O segundo nível é definido na negociação com uma Direção Participativa por Objetivos com os serviços assistenciais responsáveis pelos resultados, o que leva a uma descentralização por centros de responsabilidade do orçamento destinado, que permite envolver os profissionais nos processos de tomada de decisão.

O Consórcio Sanitário é uma fórmula orgânica de colaboração entre diferentes Administrações, ainda que também possam fazer parte dele entidades privadas sem fins lucrativos, sem que com isso se desvirtue a natureza pública da entidade. A criação dos consórcios sanitários justifica-se pela necessidade de se encontrar respostas às necessidades concretas de ordenação e de otimização de recursos em um território determinado.

Mecanismo de Gestão Indireta é caracterizado pela produção do serviço público mediante a contratação de entidades geralmente privadas com ou sem fins lucrativos. As modalidades de gestão apro- fundam a separação de funções e as relações contratuais entre as partes. Destacam-se as Entidades de Base Associativa (Sociedades Anônimas), que foram implantadas de forma experimental na Catalunha e caracterizam-se por dispor de personalidade jurídica própria e serem controladas majoritariamente por profissionais de saúde da Atenção Primária. As relações com o financiador são do tipo contratual, podendo dispor de um orçamento dedicado à compra de alguns produtos e a consultas externas, com mecanismos de corresponsabilidade no gasto.

A Gestão Indireta também pode utilizar-se de Acordos com provedores privados (com ou sem fins lucrativos), que têm sido bastante utilizados pelo Insalud e pela maioria dos atuais serviços de saúde na Espanha.

Todas as inovações organizacionais e de gestão podem possibilitar importantes melhorias na eficiência, conforme indicam as avaliações existentes de algumas experiências realizadas por Martín (1998) há mais de uma década. No entanto, apresentam a priori importantes riscos derivados da ausência de um marco de regulação institucional adequado e a inexistência de incentivos por parte dos principais agentes para configurá-lo. Além disso, outra questão crítica refere-se ao tipo de regulação a qual se submetem as empresas públicas, de forma que o deslocamento de riscos seja efetivo e que se possam limitar as atividades de influência e interferência política. 0 caráter político da prestação de serviços sanitários, a existência de um marco geral normativo homogêneo, claro e explícito de regulação das empresas públicas sanitárias e as novas formas de gestão (públicas e privadas) possibilitam altos custos de influência política.

\section{o Conselho Interterritorial e o Planejamento}

O Conselho Interterritorial do Sistema Nacional de Saúde (CISNS) é o órgão de coordenação, organismo permanente de coordenação, cooperação, comunicação e informação dos serviços de saúde entre eles e com a Administração do Estado, e tem a finalidade de promover a coesão do Sistema Nacional de Saúde através da garantia efetiva dos direitos dos cidadãos em todo o território do Estado (World Health Orga- 
nization, 2002). É composto de um total de trinta e quatro membros, dos quais a metade representa a Administração Central (Ministério da Saúde) e a outra metade as CCAA. No CISNS as decisões devem ser adotadas por consenso e apontar recomendações ou, em alguns casos, as CCAA e a Administração Central podem firmar convênios/acordos que obrigam o cumprimento das responsabilidades assumidas por ambas as partes.

Do ponto de vista do planejamento, desde o início dos anos 1990, tanto o nível central quanto os níveis regionais preparam seus próprios Planos de Saúde, com ampla mobilização social, os quais devem ser aprovados pelos respectivos parlamentos. A essas instâncias governamentais cabe buscar um consenso que assegure a aprovação dos vários Planos de Saúde conforme as prioridades sanitárias e não somente atenda aos interesses das diferentes forças políticas. A relevância dada aos processos de planejamento regional foi resultado de dois processos políticos distintos: a introdução das metas "saúde para todos" pela OMS na legislação espanhola em 1990, promovida por políticos e profissionais da saúde relacionados às áreas de epidemiologia e saúde pública, que buscavam assegurar e ampliar a qualidade, a equidade e a efetividade clínica no sistema de saúde; e as discussões iniciadas ainda nos anos 1980 sobre medidas de contenção de gastos em saúde, as quais procuravam estabelecer prioridades como uma estratégia de controlar o aumento da demanda e consumo de serviços sanitários (World Health Organization, 2002).

A experiência do funcionamento do Sistema Nacional de Saúde como sistema descentralizado desde o início dos anos 1980 até os dias atuais tem sido avaliada como positiva pelo Relatório Anual do Sistema Nacional de Saúde (España, 2008a). A qualidade dos serviços tem melhorado a mobilidade dos pacientes e sua livre circulação tem sido garantida, considerando-se, portanto, que há uma boa coordenação no âmbito das ações e planejamento em saúde pública. O relatório enfatiza, contudo, que há tensões de diversas ordens que apontam a necessidade de um esforço constante no sentido de estabelecer mecanismos de diálogo e respeito institucional e a importância de fortalecer o papel de coordenação do Conselho Interterritorial para o estabelecimento de políticas de saúde que atendam realmente às necessidades do país.

\section{o Sistema de Financiamento}

Urbanos e Utrilla (2001) apontam que, de acordo com a normativa atual, o financiamento dos serviços de assistência é descentralizado e segue o modelo de descentralização da gestão sanitária. As CCAA elaboram anualmente o orçamento, que deve conter minimamente gastos e investimentos estabelecidos no sistema de financiamento autonômico. $\mathrm{O}$ orçamento, uma vez aprovado, deve ser remetido aos órgãos competentes da Administração do Estado, para fins de avaliação da aplicação do financiamento total que se destina à assistência sanitária. Consequentemente, o financiamento e o gasto sanitário das CCAA adquirem um papel bastante relevante dentro do Sistema Nacional de Saúde num contexto de descentralização tão importante como o atualmente existente. Nesse sentido, apontam os autores, o crescimento do gasto sanitário total nos últimos quinze anos é uma manifestação do caráter prioritário concedido a essa área da ação de governo em todos os níveis das Administrações Públicas. Assim, desde a década passada, o gasto sanitário vem crescendo em maior medida que a economia espanhola, especialmente pela evolução do gasto com medicamentos.

Devido à histórica autonomia das comunidades que compõem o território espanhol, o sistema de financiamento das CCAA apresenta duas modalidades distintas - País Vasco e Navarra -, e o restante do país segue uma mesma normativa constitucional, que se baseia nas seguintes fontes:

- impostos cedidos total ou parcialmente pelo Estado e outras participações na arrecadação estatal;

- impostos, taxas e contribuições estaduais (ou seja, arrecadações das próprias CCAA);

- transferências de um Fundo de Compensação Interterritorial e outras contribuições com encargo aos Orçamentos Gerais do Estado;

- recursos procedentes de seu patrimônio e de contratos de direito privado;

- produto de operações de crédito.

A Lei Orgânica de Financiamento das Comunidades Autônomas (LOFCA), com suas sucessivas 
reformas e outras leis e acordos posteriores, desenvolveram um sistema de financiamento cujos recursos mais importantes estão constituídos por distintas receitas que garantem o desenvolvimento da autonomia financeira regional. 0 novo sistema de financiamento introduziu mudanças substanciais no modelo estabelecido, tendo em vista que integra o financiamento da saúde e dos serviços sociais com o restante das competências do Estado.

O modelo vigente estabelece um valor mínimo que as CCAA devem destinar anualmente para financiar as questões sanitárias de acordo com a evolução geral do crescimento do país. Esse sistema substituiu o critério de divisão inicial de recursos com as CCAA, baseado unicamente na cobertura populacional, por outro critério per capita, ajustado pela população maior de 64 anos, bem como um critério de risco. Isso supõe um maior ajuste entre o financiamento e as necessidades de gasto, dada a incidência demonstrada de ambas variáveis - idade e risco - nos custos da assistência sanitária.

Assim, a distribuição dos recursos segue as seguintes proporções: a população protegida consome em torno de $75 \%$ dos recursos, os maiores de 65 anos consomem cerca de $24,5 \%$ e a variável risco fica por volta de $0,5 \%$, com variações significativas conforme a região. Além disso, o novo modelo de financiamento incorpora um mecanismo transitório de garantia da suficiência dinâmica, pelo qual o governo garantiu durante os três primeiros anos (2002, 2003 e 2004) que o gasto sanitário crescesse no mínimo no mesmo ritmo que o PIB nominal.

Os resultados iniciais do modelo vigente apresentam, conforme Urbanos e Utrilla (2001), uma margem financeira importante em termos de suficiência no curto prazo. Os recursos complementares advindos da aplicação das variáveis de divisão proporcional e as cláusulas de garantias, juntamente com o processo de negociação das transferências sanitárias, permitem garantir a cobertura das necessidades de gasto. No entanto, as diferenças regionais apresentaram acréscimo importante devido, principalmente, à incidência de outras variáveis que não foram contempladas na divisão proporcional estabelecida até o presente momento e aos resultados do processo de negociação do repasse das competências sanitárias. Dessa forma, as regiões que apresentam maiores PIB per capita também apresentaram ganhos maiores com o novo modelo (Aragón, La Rioja), enquanto outras regiões menos desenvolvidas apresentaram menores recursos para investimentos em saúde (Andaluzia, Múrcia e Comunidade Valenciana).

\section{Perspectivas para a Sustentabilidade e Desafios Emergentes}

O Sistema Nacional de Saúde espanhol apresenta uma grande resolutividade e capacidade de resposta, porém algumas questões apontam a necessidade de investimentos e constituem novos desafios para todos os envolvidos em manter e ampliar a qualidade da atenção à saúde e de vida das pessoas que vivem nesse país. Um primeiro desafio mencionado no Relatório Anualcuja elaboração contou com especialistas em temas específicos e apresentou resultados de pesquisas realizadas com a população por meio de um instrumento denominado "Barômetro Sanitário", revela que o crescimento relativo populacional tem influenciado o equilíbrio da balança ofertademanda por assistência sanitária nos últimos cinco anos. Isso se deve ao fenômeno da imigração, de acordo com o Instituto Nacional de Estatística (España, 2008a), cujo crescimento somente entre 2002 e 2003 registrou 2,1\%, porém vem decrescendo no último biênio devido às medidas restritivas adotadas. A legislação espanhola sobre proteção à saúde é considerada uma das mais avançadas da Europa, tendo em vista que todos os imigrantes sem visto de permanência têm direito à assistência de urgência e menores ou gestantes usufruem de proteção completa.

Outra questão refere-se aos residentes ocasionais e de longa duração procedentes de países europeus que também utilizam os serviços públicos de saúde sem que estejam atualizados os mecanismos de reciprocidade com seus países de origem para efeitos contributivos. Além disso, o aumento da proporção de pessoas idosas, por um lado, reflete a melhoria das condições de vida e a contribuição do setor sanitário ao bem-estar dos cidadãos espanhóis e, por outro, influencia na demanda visto que essas pessoas requerem maior atenção e cuidado. 
Paralelamente ao aumento da população, cresce a demanda por melhorias na qualidade da atenção e a exigência de incorporação das possibilidades que a ciência e o desenvolvimento tecnológico dispõem. No entanto, não está sendo possível acompanhar o aporte de recursos necessários para essa incorporação na velocidade da oferta de novos produtos e medicamentos disponíveis, embora o aumento do gasto no setor se deva, em grande medida, pelo aumento dos gastos com medicamentos. Ao contrário, o gasto sanitário público está paralisado em torno de 5,4\% do PIB nos últimos anos, o que é incompatível com o crescimento da demanda e o encarecimento dos custos-saúde.

Ao comparar a porcentagem do gasto sanitário público com o gasto sanitário total, observa-se que a Espanha apresenta uma proporção sensivelmente menor em relação aos outros países da União Europeia (Eurostat, 2007). Entre 1992 e 2002, ocorreu um forte aumento do peso do setor privado, que cresceu de $22,6 \%$, sobre o gasto total, para $28,6 \%$. Pode-se observar como o peso relativo dos financiamentos público e privado da saúde se mantém relativamente estável, crescendo ligeiramente o peso da saúde privada como uma tendência na Europa.

Outro fator considerado desafio para a sustentabilidade do SNS espanhol diz respeito à insatisfação salarial dos profissionais da saúde, principalmente os médicos, devido ao congelamento salarial, ao aumento das demandas assistenciais sem a revisão dos acordos estabelecidos e uma parcela, todavia, segue em regime de contrato temporário de trabalho, o que foi corrigido com o processo de transferência para as CCAA. Somado a estes fatores há falta de determinados especialistas em regiões específicas, 0 que aponta para a necessidade de planejamento das necessidades atuais e futuras (Durán e col., 2006).

A opinião pública percebe essas tensões, o que se traduz numa piora da percepção e da valorização dos serviços de saúde, revelados no decréscimo dos índices agregados de satisfação dos usuários medidos pelo Observatório do Sistema Nacional de Saúde (2007). Este apontou o tempo de espera, especialmente para a atenção especializada, como o principal fator de insatisfação, bem como a falta de informação nas consultas em todos os níveis assistenciais, a informação recebida sobre direitos e instrumentos de reclamação e denúncia.
Esses são os principais desafios que emergem no cenário atual do Sistema Nacional de Saúde espanhol. Vale ressaltar que a saúde representa um valor social compartilhado pelos cidadãos e que as autoridades nacionais e locais elegem-na prioridade na agenda política do país, o que foi reafirmado em recente documento intitulado "Pacto por la Sanidad" (España, 2008b), cujo propósito é estabelecer prioridades políticas conjuntas entre os diferentes níveis de gestão do sistema de saúde, procurando garantir e reforçar a qualidade e a sustentabilidade do Sistema Nacional de Saúde (SNS). No documento foram apresentados seis comitês institucionais que deverão elaborar propostas consensuadas com diferentes grupos representantes das sociedades médicas, de coletivos profissionais da saúde, das sociedades científicas, da sociedade civil organizada, dos membros de sindicatos, de deputados e senadores e da representação de usuários. Esse amplo foro intenta reunir e promover a participação de distintos atores sociais para definir políticas de saúde no país, o que aponta a convergência de esforços no sentido do enfrentamento das novas exigências da contemporaneidade.

\section{Referências}

ALMEIDA, C. M. As reformas sanitárias nos anos 80: crise ou transição? 1995. Tese (Doutorado em Saúde Pública) - Escola Nacional de Saúde Pública da Fundação Oswaldo Cruz, Rio de Janeiro, 1995.

DEL AGUILA, R.; MONTORO, R. El discurso político de la transición española. Madrid: Siglo XXI, 1984 .

DURÁN, A.; LARA, J. L.; VAN WAVEREN, M. Spain: health system review, Health Systems in Transition, Copenhagen, v. 8, n. 4, p. 1-208, 20o6. Disponível em: <http://www.euro.who.int/ Document/E89491.pdf>. Acesso em: 14 abr. 2008.

ESPAÑA. Ministerio de Sanidad y Política Social. Informe SNS 2005. Madrid, 2007. Disponível em: <http://www.msc.es/ organizacion/sns/informeAnualSNS/docs/2005/ capiInformeSNS2005.pdf >. Acesso em: 14 jul. 2008. 
ESPAÑA. Instituto Nacional de Estadística. Indicadores sociales de España 2007. Madrid, 2008a. Disponível em: <http://www.ine.es/prodyser/ pubweb/indisoco3/indisoco3_tab.pdf $>$. Acesso em: 14 jul. 2008.

ESPAÑA. Ministerio de Sanidad y Consumo. Pacto por la sanidad. Madrid, 2008b. Disponível em: <http://www.msps.es/novedades/evento/pactoSNS. htm\#pilar>. Acesso em: 10 mar. 2010.

ETVITI, J.; LEYVA, R. F. Social and political actors in the formulation of health reform in Spain. Cadernos de Saúde Pública, Rio de Janeiro, v. 11, n. 1, p. 34-44, 1995 .

FERNÁNDEZ-CUENCA, R. G. Análisis de los servicios sanitarios. In: CATALÁ, F. J.; KEENOY, E. M. Informe SESPAS 1998: la salud pública y el futuro del estado del bienestar. Granada: Escuela Andaluza de Salud Pública, 1998. p. 251-298.

GIL, A. C. Métodos e técnicas de pesquisa social. 4. ed. São Paulo: Atlas, 1994.

MARCONI, M. A.; LAKATOS, E. M. Fundamentos de metodologia científica. 6. ed.São Paulo: Atlas, 2008.

MARTíN, J. J. M. Riesgos y oportunidades de las empresas públicas sanitárias y nuevas formas organizativas y de gestión. In: SILIÓ, F. V.; KEENOY, E. M. La gestión del cambio en los servicios sanitarios. Granada: Escuela Andaluza de Salud Pública, 1998. p. 85-106.

ORTÚN, V. R. Sistema sanitario y estado del bien estar: problemas a la vista. In: CATALÁ, F. J.; KEENOY, E. M. Informe SESPAS 1998: la salud pública y el futuro del estado del bienestar. Granada: Escuela Andaluza de Salud Pública, 1998. p. 40-59.
PONSÁ, J. et al. Evaluación de la reforma de la atención primaria y de la diversificación de los proveedores sanitarios, Fulls econòmics del sistema sanitari. Barcelona, n. 37, setembre, 2003. REY DEL CASTILLO, J. Formas de descentralización de los servicios sanitarios. In:___. Descentralización de los servicios sanitarios: aspectos generales y análisis del caso español. Granada: Escuela Andaluza de Salud Pública, 1998. p. 41-91. (Série Monografias, 23). URBANOS, R. M.; UTRILLA, A. El nuevo sistema de financiación sanitaria y sus implicaciones económicas en las Comunidades Autónomas. Revista de Administración Sanitaria, Madrid, v. V, n. 20, p. 25-48, oct./dic. 2001.

VAN MOSSEVELD, C.; KAWIORSKA,D.; DE NORRE, B. Population and social conditions: Health expenditure, 2003-2005. Luxembourg: Eurostat, 2008. Disponível em: <http://epp.eurostat. ec.europa.eu/cache/ITY_OFFPUB/KS-QA-o8-026/ EN/KS-QA-o8-026-EN.PDF>. Acesso em: 10 out. 2008.

VENTURA J. V. Organización y gestión de la atención sanitaria. In: CATALÁ, F. J.; KEENOY, E. M. Informe SESPAS 1998: la salud pública y el futuro del estado del bienestar. Granada: Escuela Andaluza de Salud Pública, 1998. p. 288-362.

WORLD HEALTH ORGANIZATION. Health care systems in transition: Spain. Copenhagen: World Health Organization Regional Office for Europe, 2002.

Recebido em: $11 / 12 / 2008$

Reapresentado em: 13/08/2009

Aprovado em: 28/08/2009 